Dhaka Univ. J. Biol. Sci. 23(1): 27-38, 2014 (January)

\title{
COMMUNITY STRUCTURE ANALYSIS OF ENDOPARASITIC HELMINTHS OF ANABAS TESTUDINEUS FROM UNPOLLUTED AND POLLUTED SITES OF A FRESHWATER BODY OF DHAKA
}

\author{
Md. Osman Ghani, Aminul Islam Bhuiyan* and Jannatul Bushra \\ Department of Zoology, University of Dhaka, Dhaka-1000, Bangladesh
}

Key words: Endoparasite, Anabas testudineus, Community structure, Polluted, Unpolluted sites, Fresh water

\begin{abstract}
Community structure of metazoan endoparasites of Anabas testudineus collected from an unpolluted and a polluted water body was determined. Out of $100 \mathrm{~A}$. testudineus observed, $78 \%$ was parasitized by at least one species of endohelminth parasite. Eight metazoan endoparasites were recorded. Nematode showed higher prevalence than trematode. One species was accounted as satellite species and others as secondary. Larger hosts were more abundantly infected than the smaller ones as positive correlation was observed between the standard length of the hosts and the abundance/ prevalence of all parasites. Maximum parasite species followed a typical overdispersed type of distribution pattern. Parasite species richness was lower in fish from polluted (4) than unpolluted water (6). Per cent similarity of infestation between the sample hosts was high (55.73\%). Three common parasite species from both sample hosts showed insignificant difference in intensity of infestation in relation to host habitat. Host sex had influenced infestation at infra-community but not at component community level. Interspecies association between parasites was strong in fish from unpolluted in comparision from polluted water. Host's habitat quality also played an influential role in building parasite community, diversity and other associated community indices.
\end{abstract}

\section{Introduction}

Aquatic pollution is still a problem in many freshwater and marine environments. Fish generally harbor a wide range of ecto- and endoparasites. The later as well as their hosts might be affected in a number of ways by contaminants. Toxic chemicals have a consistent negative effect on helminths. Pollution and other man-made alterations of the aquatic environment may affect a parasite community directly by acting on free-living parasitic stages or indirectly by acting on the intermediate or the definitive host population ${ }^{(1)}$. Uptake and accumulation of pollutants by fish may bring changes in hormone levels of fish that ultimately affects on general metabolism of the associated fish parasite $^{(2)}$. In addition to effects on free-living organisms, pollutants also affect the health

*Author for correspondence: <aminul_islam89@yahoo.com>. 
of parasites and consequently their occurrence and distribution. Thus, pollutants might influence, directly or indirectly, the prevalence, intensity and pathogenicity of a parasite community. Pollutants might promote increased parasitism in aquatic animals, especially fish by impairing its immune response or favoring the survival and reproduction of the intermediate hosts ${ }^{(3)}$. Chronic exposure to pollutants may cause physiological, behavioral and biochemical changes in host that ultimately can influence the prevalence and intensity of parasitism. Additionally, if infected hosts are differentially killed by pollution, the parasite population will decline, further reducing prevalence. ${ }^{(4)}$ In contrast to toxic pollutants, eutrophication and thermal effluent often raise rates of parasitism in aquatic systems because the associated, increased productivity can increase the abundance of intermediate hosts ${ }^{(5)}$. Some authors advocate differences in composition and structure of fish parasite communities (species richness and diversity) in freshwater environments with different pollution levels as useful indices, and usefulness of parasite community structure as parameters for determining environmental quality ${ }^{(6)}$. Therefore, it can be expected that parasites may display individual, population and community level alterations in polluted than to unpolluted environments.

Investigation of pollution effect on parasitic communities in aquatic environment is scarce in Bangladesh. Recently, increasing interest is being observed among scientists in this field for their awareness about environmental pollution and its negative impacts on aquatic biota as well as to our own. Parasites can also be used as an indicator for getting information about environmental pollution. Ghani and Bhuiyan ${ }^{(7)}$ worked on the community structure of endoparasitic helminths of Channa punctatus from a freshwater river and a polluted lagoon of Bangladesh. Therefore, the present work was undertaken to determine the role of aquatic pollution in building parasite community of Anabas testudineus, collected from two sites of the same water body varying in water qualities.

\section{Materials and Methods}

The host Koi fish, Anabas testudineus (Anabantidae), was sampled from two different sites of same water body near Dhaka, selected according to their differences in water qualities as polluted water: Taiwabpur beel (TA), which receives effluents directly from nearby industries, Ashulia, Dhaka $\left(23^{\circ} 53^{\prime} \mathrm{N}-90^{\circ} 24^{\prime} \mathrm{E}\right)$; and unpolluted water: Beribadh point of Turag river, Diabari, Mirpur (DM), Dhaka $\left(23^{\circ} 52^{\prime} \mathrm{N}-90^{\circ} 24^{\prime} \mathrm{E}\right)^{(8)}$. The later water body is regularly flushed out with water from upstream.

A total of 100 (50 from each site) A. testudineus were collected from the local fishermen in between April and October, 2012. Live fishes were immediately brought to the Parasitology Laboratory of the Department of Zoology, University of Dhaka.

The examination was aimed at studying the infection in the fish by metazoan endoparasitic helminths. Standard length and sex of each fish were noted before autopsy. 
Sex, size and organwise infections by each species of parasite were recorded. Berland' $\mathrm{s}^{(9)}$ methods were used for staining and mounting of parasites.

The methods used here to describe general features of parasite infra-communities (prevalence, abundance, and intensity) and component community. Component community structure of parasite was described by traditional descriptors (e.g. species richness, mean abundance and mean intensity) and community similarity. To describe the structure of the infra- and component communities, data were related to parasite abundance, prevalence and intensity were calculated according to Bush et al.(10)

The classification of the species was done according to the importance value for the community as Central species $=$ present in more than $66.6 \%$; Secondary species $=$ present between 33.3 and $66.6 \%$ and Satellite species = lower than $33.3 \%$ of the total number of fish analyzed ${ }^{(11)}$.

Green's dispersion index (GI) and index of dispersion (DI) were used to evaluate the dispersion pattern of parasite species. Green's index takes a value of 1.0 at maximum clumping, 0 for random distribution and -1 for a perfectly uniform distribution. Dispersion index was tested using statistical ' $d$ ' as most of the parasite species did not follow theoretical Poisson distribution series. Distribution of parasites was classified as aggregated when $\mathrm{d}>1.96$; regular when $\mathrm{d}<-1.96$ and random when $\mathrm{d}<1.96$.

Simpson proposed the diversity index in ecology to measure the diversity of the species in a community. Simpson's index $(\lambda)$ and diversity are negatively related. For infinite population the Simpson's unbiased estimator of ' $\bar{\lambda}$ ' was calculated. Shannon's index of diversity $\mathrm{H}^{\prime}$ is most widely used index in community ecology. It measures the average degree of "uncertainty" in predicting to which species an individual chosen at random from a collection of 'S' species and ' $n$ ' individuals will belong to.

The density of community depends on the number of species and the evenness. So an attempt was made to quantify the evenness component of the parasite diversity in both communities. Heip proposed evenness index (Eh) and Hill's evenness index (Ен) were counted. Eн converges to one as diversity of community decreases i.e. one species tends to dominant. Modified Hill's ratio $\left(\mathrm{E}^{\prime} \mathrm{H}\right)$ is preferred over $\mathrm{EH}_{\mathrm{H}}$ as it has a clearly desirable property for an evenness index. Though there are various evenness index, Ен and $\mathrm{E}^{\prime} \mathrm{H}$ are relatively unaffected by species richness and they also remain consistent with sample variation. Hence we mainly considered the computed value of $\mathrm{Eн}_{\mathrm{H}}$ and $\mathrm{E}_{\mathrm{H}}^{\prime}$ in present study.

Pearson linear correlation coefficient ( $\mathrm{r}$ ) and Spearman's rank correlation coefficient $\left(r_{s}\right)$ were computed to determine possible correlations between the host's standard length with the prevalence and abundance of each parasite species, respectively.

Jaccard's index (JI) was used to compare the presence/absence of two parasite species occurring in the same host at a time. It always contains a value between 0 to 1 and as the value approaches to one, indicates the association between species is strong. Per cent 
similarity compared the mean abundances of the fish parasite component communities in each site was computed.

Kruskal-Wallis test was performed to compare the influence of habitats on parasitism levels. For determining the probable variation of diversity in relation to host sex, MannWhitney ' $U$ ' test was conducted. Since the experimental data showed abnormal distribution the above testes were applied.

Statistical tests were conducted by using XLSTAT 2011 (Kruskal-Wallis test) and SPSS 16.0 (Pearson linear correlation, Spearman's rank correlation and Mann-Whitney ' $U$ ' test) software package. Statistical significance level was adopted at $\mathrm{p} \leq 0.05$.

\section{Results and Discussion}

Altogether, eight metazoan endoparasites were recorded during the present study. The parasites recorded were two digenetic trematode identified as Allocreadium minutum and Neopecoelina saharanpuriensis, six nematodes, viz. Ascaridida sp., Gnathostoma spinigerum, Contracaecum sp., Camallanus anabantis, Camallanus pearsei (both species were considered as Camallanus spp. during calculation) and Paragendria wallagonia. Of the eight parasites N. saharanpuriensis, Ascaridida sp. and Contracaecum sp. were common in both sites. A. minutum, N. saharanpuriensis, Ascaridida sp., G. spinigerum, Contracaecum sp., P. wallagonia were recorded from fishes of unpolluted site and $N$. saharanpuriensis, Ascaridida sp., Contracaecum sp., C.anabantis, C. pearsei from the fishes of polluted site.

In the present study, 72 and $84 \%$ fishes were infested with at least one species of parasites from unpolluted (DM) and polluted (TA) sample, respectively. Prevalence of infestation varied with water quality and sex of host. In both the samples, female (DM = $81.25 \%, \mathrm{TA}=90 \%)$ hosts were more infested than male $(\mathrm{DM}=55.75 \%$, $\mathrm{TA}=75 \%)$. Host

sex can influence parasitism levels due to the behavior and physiological differences between the sexes ${ }^{(12)}$. This might be due to the physiological state of the females, as most gravid females could have reduced resistance to infection by parasites. In addition, their increased rate of food intake to meet their food requirements for the development of their egg might have exposed them to more contact with the parasites, which subsequently increased their chance of being infected.

In the host body, the most preferred habitat for these parasites was intestine. All parasites of DM fishes were intestinal inhabitants besides three nematode larvae were also in stomach. In TA site, all parasites were found in intestine of hosts while two nematodes were also in stomach (Table 1). The site segregation of the endoparasites of $A$. testudineus found in the present study is probably due to the mechanism of niche restriction, infection with different parasites at different times of the year or potential mechanism to avoid competition involved for the constituent parasite community. Digenean parasites feed mainly on mucosa and epithelial tissue, therefore, they will be browsing in the intestine, in places where the epithelial turnover rate is high ${ }^{(13)}$. The fact 
that the two digenean parasites in our study were most frequently found in the posterior part of the gut could support this hypothesis. Nematodes are equipped with an alimentary canal and would thus be free to roam around the host's alimentary tract. The presence of some nematode larvae in the stomach might be made possible by their possession of a thick cuticle, which enables them to withstand the harsh conditions or they were on route for completing life cycle via stomach.

Table 1. Location of infection (LI), developmental stages (DS), number of infected fish (IF), number of parasite (NP) and ranges of parasite from the specimens of A. testudineus from two sampling sites.

\begin{tabular}{|c|c|c|c|c|c|c|c|c|c|c|}
\hline \multirow{2}{*}{ Name of parasite } & \multicolumn{5}{|c|}{ Diabari, Mirpur (DM) } & \multicolumn{5}{|c|}{ Taiwabpur, Ashulia (TA) } \\
\hline & LI & DS & IF & $\mathrm{NP}$ & Ranges & LI & DS & IF & $\mathrm{NP}$ & Ranges \\
\hline Allocreadium minutum & I & $\mathrm{A}$ & 26 & 61 & $1-4$ & - & - & - & - & - \\
\hline Neopecoelina saharanpuriensis & I & $\mathrm{A}$ & 20 & 34 & $1-3$ & I & A & 20 & 34 & $1-3$ \\
\hline Ascaridida sp. & S, I & $\mathrm{L}$ & 22 & 51 & $1-5$ & S, I & $\mathrm{L}$ & 16 & 44 & $1-7$ \\
\hline Gnathostoma spinigerum & $S, I$ & $\mathrm{~L}$ & 21 & 38 & $1-2$ & - & - & - & - & - \\
\hline Contracaecum sp. & S, I & $\mathrm{L}$ & 23 & 46 & $1-3$ & S, I & $\mathrm{L}$ & 24 & 64 & $1-3$ \\
\hline Paragendria wallagonia & I & $\mathrm{A}$ & 14 & 28 & $1-2$ & & & & & \\
\hline Camallanus spp. & - & - & - & - & - & I & $\mathrm{A}$ & 15 & 32 & $1-4$ \\
\hline
\end{tabular}

$\mathrm{I}=$ intestine, $\mathrm{S}=$ stomach, $\mathrm{L}=$ larva, $\mathrm{A}=$ adult.

Parasite communities in sampled fish were composed of digenetic trematode and nematode endoparasites only. It was observed that infestation with trematode was higher in unpolluted $(62 \%)$ than polluted site $(48 \%)$ fishes. Infestation with nematode was slightly higher in polluted (72\%) than unpolluted (70\%) site fishes. Average parasite burden with trematode of DM sample (3.06) was higher than TA sample (2.08); on the other hand, the average parasite burden with nematode was higher in TA sample (4.09) than DM sample (3.89). There are increasing evidences that environmental pollution can affect parasite abundance and prevalence. MacKenzie et al. ${ }^{(14)}$ supports the view that there is a relation between parasite load in fish and fish exposure to a variety of pollutants. Lower number of trematode species in TA sample with low prevalence than DM might have occurred, because trematode may contact and absorb pollutants from two sources, i.e. from host tissues during endoparasitic phases of their life cycles, and/or directly from the environment during the relatively brief periods in which they are freeliving. Low prevalence of trematode in polluted site near industrial areas ecosystem that suffer great anthropic pressure resulting from organic and industrial discharges, which subsequently leads the recruitment process unsuccessful for trematode as it follows a complex life cycle. However, presence of the nematodes in the polluted sites may make possible by their possession of a thick cuticle, which enables them to withstand the harsh conditions. 
According to status of distribution, Camallanus spp. accounted as satellite in TA sample and other species from both sites were considered as secondary; no central species found. Mean intensity was higher for all parasites of TA than DM sample (Table 2). Different hosts utilize different parts of their environment, then this will often lead to heterogeneities in parasite intensities across the population of hosts. Higher prevalence and intensity of parasitic infection was recorded from TA sample and this might be due to immune suppressor toxins and heavy metal present in that aquatic environment which directly or indirectly decreased the host immune system and increased susceptibility of host population to parasitic infection ${ }^{(3)}$.

Table 2. Prevalence (P\%), mean intensity (MI), mean abundance (MA) and status of species of the helminth endoparasite community of $A$. testudineus from two collection sites.

\begin{tabular}{|c|c|c|c|c|c|c|c|c|}
\hline \multirow{2}{*}{ Name of parasite } & \multicolumn{4}{|c|}{ Diabari, Mirpur (DM) } & \multicolumn{4}{|c|}{ Taiwabpur, Ashulia (TA) } \\
\hline & $\mathrm{P} \%$ & $\mathrm{MA} \pm \mathrm{SD}$ & $\mathrm{MI} \pm \mathrm{SD}$ & Status & $\mathrm{P} \%$ & $\mathrm{MA} \pm \mathrm{SD}$ & $\mathrm{MI} \pm \mathrm{SD}$ & Status \\
\hline A. minutum & 46 & $1.22 \pm 1.56$ & $2.65 \pm 1.19$ & Secondary & - & - & - & - \\
\hline N. saharanpuriensis & 40 & $0.68 \pm 0.98$ & $1.70 \pm 0.73$ & $"$ & 46 & $0.94 \pm 1.15$ & $2.04 \pm 0.77$ & Secondary \\
\hline Ascaridida sp. & 44 & $1.02 \pm 1.50$ & $2.32 \pm 1.46$ & $"$ & 36 & $0.88 \pm 1.29$ & $2.44 \pm 0.86$ & $"$ \\
\hline G. spinigerum & 50 & $0.76 \pm 0.85$ & $1.52 \pm 0.51$ & $"$ & - & - & - & - \\
\hline Contracaecum sp. & 60 & $0.92 \pm 0.99$ & $1.53 \pm 0.82$ & $"$ & 50 & $1.28 \pm 1.44$ & $2.56 \pm 0.92$ & Secondary \\
\hline Camallanus spp. & - & - & - & - & 28 & $0.64 \pm 1.14$ & $2.28 \pm 0.91$ & Satellite \\
\hline P. wallagonia & 44 & $0.56 \pm 0.70$ & $1.27 \pm 0.46$ & Secondary & - & - & - & - \\
\hline
\end{tabular}

Parasites from both samples had shown typical over dispersed type of distribution pattern for most of the cases. In DM sample two species showed aggregated $(\mathrm{d}>1.96)$ and others suggested their random distribution pattern $(\mathrm{d}<1.96)$. In case of TA sample Camallanus spp. showed the highest dispersion index value $(\mathrm{DI}=2.031)$ and other species had aggregated distribution except $N$. saharanpuriensis $(\mathrm{d}<1.96)$, which showed random pattern of distribution (Table 3). Aggregated distribution of the parasite population is considered as one of the most common features of metazoan parasite infections ${ }^{(15)}$. Most of the species of endoparasites from TA sample analyzed presented a spatial aggregate pattern in agreement to the typical patterns of endoparasitism showed by Oliva et al.(16)

Diversity indices indicated that diversity of species was different in two sites. Simpson's index $(\lambda)$ was lower in DM sample $(\lambda=0.178)$ than that of TA sample $(\lambda=$ 0.265); which indicated that DM parasite community was well diverse than TA community. Simpson's unbiased estimator $(\bar{\lambda})$ and Shannon's index $\left(\mathrm{H}^{\prime}\right)$ also showed that the parasite community from DM was well diverged than the TA fishes (Table 4). Generally, parasite species diversity in healthy ecosystems, where most hosts and vectors are present, is richer than in polluted ecosystems. For that reason, DM sample was more divergent with increasing species richness (6) than that of TA sample (4). Heip evenness index and Hill's evenness index, showed minute difference in TA $\left(\mathrm{E}_{\mathrm{h}}=0.962, \mathrm{EH}_{\mathrm{H}}=0.971\right)$ than $\mathrm{DM}\left(\mathrm{E}_{\mathrm{h}}=0.960, \mathrm{E}_{\mathrm{H}}=0.968\right)$ sample fishes, meant that community structure was 
constructed by evenness with their constituent parasite community in each of the sampled fishes. It was also observed that per cent similarity between the samples gained a higher value $(55.73 \%)$ which indicated that both samples shared similar species at great

Table 3. Values of variance to mean ratio (DI), Green's aggregate index (GI) and statistical ' $d$ ' of the metazoan endoparasites of $A$. testudineus from DM and TA samples.

\begin{tabular}{|c|c|c|c|c|c|c|c|c|}
\hline \multirow{2}{*}{ Name of parasite } & \multirow[b]{2}{*}{ DI } & \multicolumn{3}{|c|}{ Diabari, Mirpur (DM) } & \multicolumn{4}{|c|}{ Taiwabpur, Ashulia (TA) } \\
\hline & & GI & $\mathrm{d}$ & Distribution type & DI & GI & $\mathrm{d}$ & Distribution type \\
\hline A. minutum & 1.984 & 0.016 & 4.095 & Aggregated & - & - & - & - \\
\hline N. saharanpuriensis & 1.412 & 0.012 & 1.914 & Random & 1.407 & 0.009 & 1.894 & Random \\
\hline Ascaridida sp. & 2.206 & 0.004 & 4.854 & Aggregated & 1.891 & 0.021 & 3.764 & Aggregated \\
\hline G. spinigerum & 0.951 & -0.001 & -0.195 & Random & - & - & - & - \\
\hline Contracaecum sp. & 1.065 & 0.001 & 0.367 & $"$ & 1.620 & 0.010 & 2.751 & Aggregated \\
\hline Camallanus spp. & - & - & - & - & 2.031 & 0.033 & 4.259 & $"$ \\
\hline P. wallagonia & 0.875 & -0.005 & -0.589 & Random & - & - & - & - \\
\hline
\end{tabular}

Table 4. Different diversity and evenness indices of helminths endoparasite component communities in A. testudineus from DM and TA sample fishes.

\begin{tabular}{lcc}
\hline Diversity indices/Evenness indices & DM & TA \\
\hline Simpson index $(\lambda)$ & 0.178 & 0.265 \\
Simpson unbiased estimator $(\overline{\bar{\lambda}})$ & 0.174 & 0.261 \\
Shannon index $\left(\mathrm{H}^{\prime}\right)$ & 1.758 & 1.357 \\
Heip evenness index $\left(\mathrm{E}_{\mathrm{h}}\right)$ & 0.960 & 0.962 \\
Hill's evenness index $\left(\mathrm{E}_{\mathrm{H}}\right)$ & 0.968 & 0.971 \\
Modified Hill's ratio $\left(\mathrm{E}^{\prime}\right.$ н) & 0.962 & 0.961 \\
\hline
\end{tabular}

number and these common parasite species also near equally distributed among fishes. Greater number of species found in DM sample probably was due to the greater number of invertebrate species fauna associated to aquatic system shared by the host that sometimes not possible for TA sample as the number of invertebrate species fauna associated to the aquatic system might less due to pollution. With reference to water $\mathrm{pH}$ modification, Marcogliese and Cone ${ }^{(17)}$ observed a reduction of species richness of parasite assemblages hosted by yellow eels sampled in sites of low $\mathrm{pH}$ within a single watershed. Similarly, Gelnar et al.(18) documented the close relationships between parasite community descriptors (species richness, diversity and relative abundance) and water pollution levels evaluated by using indices based on the invertebrate communities. Unpolluted ecological niche occupy higher diverse feeding qualities for the sampled fish due to absence of pollution, including a greater number of prey species in their diet, present them favorable conditions to higher endoparasitic infections and ultimately increase endoparasitic richness in DM sample. 
It was observed that all parasite species showed positive correlation with host's standard length. Significant positive correlation was observed between the host standard length and the prevalence and abundance for all parasite species in DM sample specimen at $p<0.05$ or $p<0.01$ level of significance. While in case of TA sample, all parasites showed insignificant positive correlation between host's standard length with the prevalence of parasite but significant positive correlation between host's standard length and the abundance for all parasite species ( $<$ 0.01) except Ascaridida sp. (Table 5). In the present study, host size was always positively correlated with the prevalence and abundance of

Table 5. Values of Pearson's correlation coefficients (r) and Spearman's rank correlation coefficients $\left(r_{s}\right)$ to evaluate the relationship between abundance and prevalence with standard length of $A$. testudineus.

\begin{tabular}{|c|c|c|c|c|c|c|c|c|}
\hline \multirow[t]{2}{*}{ Name of parasite } & \multicolumn{4}{|c|}{ Diabari, Mirpur (DM) } & \multicolumn{4}{|c|}{ Taiwabpur, Ashulia (TA) } \\
\hline & $\mathrm{r}$ & $\mathrm{p}$ & $\mathrm{r}_{\mathrm{s}}$ & $\mathrm{p}$ & $\mathrm{r}$ & $\mathrm{p}$ & $\mathrm{r}_{\mathrm{s}}$ & $\mathrm{p}$ \\
\hline A. minutum & 0.491 & $0.000^{* *}$ & 0.525 & $0.000^{* *}$ & - & - & - & - \\
\hline N. saharanpuriensis & 0.044 & $0.001^{* *}$ & 0.465 & $0.001^{* *}$ & 0.114 & 0.431 & 0.543 & $0.000^{* *}$ \\
\hline Ascaridida sp. & 0.537 & $0.000^{* *}$ & 0.631 & $0.000^{* *}$ & 0.238 & 0.096 & 0.130 & 0.369 \\
\hline G. spinigerum & 0.489 & $0.000^{* *}$ & 0.563 & $0.000^{* *}$ & - & - & - & - \\
\hline Contracaecum sp. & 0.370 & $0.008^{* *}$ & 0.349 & $0.013^{*}$ & 0.075 & 0.605 & 0.463 & $0.001^{* *}$ \\
\hline Camallanus spp. & - & - & - & - & 0.481 & 0.461 & 0.461 & $0.001^{* *}$ \\
\hline P. wallagonia & 0.393 & $0.005^{* *}$ & 0.406 & $0.003^{* *}$ & - & - & - & - \\
\hline
\end{tabular}

*Significance level $\mathrm{p}<0.05,{ }^{* *}$ Significance level $\mathrm{p}<0.01$.

the parasite species. The presence of relationship between abundance and prevalence with the host's standard length might due to the presence of relationship between endoparasitic diversity and the body length of the two host samples under analysis may indicate that their growth rate did not vary according to their habitat. This fact indicates homogeneity in their increasing of body length during their life cycle and thus permits the uniform recruitment of the species of endoparasites throughout their life. As pointed out by Poulin ${ }^{(19)}$, these patterns cannot be generalized because in many host-parasite species systems the correlations are positive but weak and insignificant. In addition, ontogenetical changes in feeding behavior might influence parasite prevalence and abundance in the different host size classes ${ }^{(20)}$.

Results of the Mann-Whitney ' $U$ ' test showed that intensity of infection and parasite abundance did not significantly $(\mathrm{p}>0.05)$ affected with host's sex (Table 6). The insignificant values with regard to host sex from Mann-Whitney test of DM and TA samples evidenced that both sample's ecological relationships (occupation of habitat and diet) are similar among males and females. Same results have been obtained for other 
species of freshwater fishes. ${ }^{(21)}$ Moreover, Poulin ${ }^{(22)}$ stated that high testosterone levels can cause immune suppression in males and could lead in some cases to males suffering more from parasites than do females, although many parasite surveys have reported no significant differences in infection abundance and prevalence between female and male hosts.

Table 6. Values of Mann-Whitney test $(\mathrm{U})$ to evaluate the role of host sex with parasite abundance in sample of DM and TA fishes.

\begin{tabular}{lcccccc}
\hline \multirow{2}{*}{ Name of parasite } & \multicolumn{2}{c}{ Diabari, Mirpur $(\mathrm{DM})$} & & \multicolumn{2}{c}{ Taiwbpur, Ashulia (TA) } \\
\cline { 2 - 3 } \cline { 5 - 6 } & $\mathrm{Z}(\mathrm{U})$ & $\mathrm{p}$ & & $\mathrm{Z}(\mathrm{U})$ & $\mathrm{p}$ \\
\hline A. minutum & -0.789 & 0.425 & & - & - \\
N. saharanpuriensis & -0.93 & 0.353 & & -0.485 & 0.628 \\
Ascaridida sp. & -1.465 & 0.143 & & 0.267 & 0.789 \\
G. spinigerum & -1.667 & 0.095 & & - & - \\
Contracaecum sp. & 1.152 & 0.249 & & -0.505 & 0.614 \\
Camallanus spp. & - & - & & -1.782 & 0.075 \\
P. wallagonia & 0.222 & 0.824 & & - & - \\
\hline
\end{tabular}

$\mathrm{p}=$ significance level.

Since we were concerned with how often two species were found in same host, Jaccard's index (JI) was used to observe the interspecific association between each pair of parasite species. From the value of JI, it could be considered that interspecific association between two species of parasite was stronger in DM sample than TA sample (Table 7). It was observed that there is no or less evidence of competition among the endoparasites in DM host sample since for most cases species to species association occurred with higher values suggesting that they have the common and continuous reservoirs/intermediate hosts which remain stable with time. In TA sample one species pair (Contracaecum sp.Camallanus spp. JI $=0.522$ ) presented a high associative degree suggesting their close association for sharing the same reservoirs/intermediate hosts. The lack of association for other endoparasite pair of TA sample suggested that the fish did not simultaneously ingest the intermediate hosts and feed different items in their diet. However, it is necessary that some factors related to the origin of associations should be taken into consideration, such as - (a) low immunological resistance of hosts with regard to endoparasites; (b) different susceptibilities of hosts, (c) similarity or difference of intermediate hosts and (d) necessary conditions for the survival of parasites ${ }^{(11)}$. These results reinforce the postulate of Rohde et al.(23) according to which the parasite community structure in marine fishes constitutes a confused and unsaturated species complex, unlike the interactive patterns related for other host groups.

Kruskal-Wallis test was conducted to evaluate the role of habitats on parasitic infestation for the three common parasites obtained from both the samples. According to 
the test, three common parasite species showed positive but insignificant difference $(p>0.05)$ of infestation in relation to host habitats (Table 8). Comparing the structure of the endoparasitic communities of the two sample hosts one may observe that they did not share the same parasite species composition even though; both of them share the same river flow.

Table 7. Values of Jaccard's index (JI) to estimate interspecific association between each pair of parasite species of A. testudineus collected from two sites of Bangladesh.

\begin{tabular}{|c|c|c|c|c|c|c|c|c|c|c|}
\hline \multirow[t]{2}{*}{ Name of parasite } & \multicolumn{6}{|c|}{ Diabari, Mirpur (DM) } & \multicolumn{4}{|c|}{ Taiwbpur, Ashulia (TA) } \\
\hline & $\mathrm{T} 1$ & $\mathrm{~T} 2$ & N1 & $\mathrm{N} 2$ & N3 & N5 & $\mathrm{T} 2$ & $\mathrm{~N} 1$ & N3 & N4 \\
\hline A. minutum (T1) & - & 0.483 & 0.665 & 0.517 & 0.519 & 0.452 & 0 & 0 & 0 & 0 \\
\hline N. saharanpuriensis (T2) & 0.483 & - & 0.556 & 0.452 & 0.515 & 0.500 & - & 0.250 & 0.485 & 0.296 \\
\hline Ascaridida sp. (N1) & 0.665 & 0.556 & - & 0.516 & 0.486 & 0.571 & 0.250 & - & 0.273 & 0.143 \\
\hline G. spinigerum (N2) & 0.517 & 0.452 & 0.516 & - & 0.618 & 0.516 & 0 & 0 & 0 & 0 \\
\hline Contracaecum sp. (N3) & 0.519 & 0.515 & 0.486 & 0.618 & - & 0.529 & 0.485 & 0.273 & - & 0.522 \\
\hline Camallanus spp. (N4) & 0 & 0 & 0 & 0 & 0 & 0 & 0.296 & 0.143 & 0.522 & - \\
\hline P. wallagonia (N5) & 0.452 & 0.500 & 0.571 & 0.516 & 0.529 & - & 0 & 0 & 0 & 0 \\
\hline
\end{tabular}

Table 8. Values of Kruskal-Wallis test $(\mathrm{H})$ of three common parasites and corresponding significance level (p) to evaluate the role of host's habitats with intensity of infestation.

\begin{tabular}{lcc}
\hline Name of parasite & $\mathrm{H}$ & $\mathrm{p}$ \\
\hline Neopecoelina saharanpuriensis & 1.014 & 0.313 \\
Ascaridida sp. & 0.294 & 0.587 \\
Contracaecum sp. & 0.442 & 0.506 \\
\hline
\end{tabular}

The study demonstrates that parasite species composition and richness in fish were influenced by local environmental factors. The results of this study indicate that the industrial and sewage effluent affects the metazoan parasite assemblage of A. testudineus. Therefore, as parasite can be used as an environmental indicator, there are several aspects of pollution-parasite research require further study in Bangladesh to assess environmental quality.

\section{References}

1. Poulin R 1992. Toxic pollution and parasitism in freshwater fish. Parasitol. Today 8: 58-61.

2. Sures B 2008. Environmental Parasitology: Interactions between parasites and pollutants in the aquatic environment. J. Helminthology 15: 434-438.

3. Khan RA and J Thulin 1991. Influence of pollution on parasites of aquatic animals. Adv. Parasitol. 30: 201-238. 
4. Stadnichenko AP, LD Ivanenko, IS Gorchenko, OV Grabinskaya, LA Osadchuk and SA Sergeichuk 1995. The effect of different concentrations of nickel sulphate on the horn snail (Mollusca: Bulinidae) infected with the trematode Cotylurus cornutus (Strigeidae). Parazitologiya 29: 112-116.

5. Marcogliese DJ 2005. Parasites of the super organism: Are they indicators of ecosystem health? Int. J. Parasitol. 82: 389-399.

6. MacKenzie K 1999. Parasites as pollution indicators in marine ecosystems: a proposed early warning system. Marine Pollution Bulletin 38: 955-959.

7. Ghani MO and AI Bhuiyan 2011. Community structure of endoparasitic helminths of Channa punctatus from a fresh water river and a polluted lagoon of Bangladesh. Bangladesh J. Zool. 39(2): 173-185.

8. Rahman AKML, M Islam, MZ Hossain and MA Ahsan 2012. Study of the seasonal variations in Turag river water quality parameters. African J. Pure and Applied Chem. 6(10): 144148.

9. Berland B 1982. Basic techniques involved in helminth preservation. In: Proceeding of Workshop on "Technology as applied to museum parasite collections." ICOPA V- 1982. Toronto, Canada p. 1-15.

10. Bush AO, KD Lafferty, JM Lotz and AW Shostak 1997. Parasitology meets ecology on its own terms. J. Parasitol. 83(4): 575-583.

11. Bush AO and JC Holmes 1986. Intestinal helminths of lesser scaup ducks: An interactive community. Can. J. Zool. 64: 142-152.

12. Esch GW, CR Kennedy, AO Bush and JM Aho 1988. Patterns in helminth communities in freshwater fish in Great Britain: alternative strategies for colonization. Parasitology 96: 519-532.

13. Crompton DWT 1973. The sites occupied by some parasitic helminths in the alimentary tract of vertebrates. Biol. Rev. 48: 27-83.

14. MacKenzie K, HH Williams, B Williams, AH McVicar and R Siddall 1995. Parasites as indicators of water quality and the potential use of helminth transmission in marine pollution studies. Advances in Parasitology 35: 85-144.

15. Poulin R 1993. The disparity between observed and uniform distributions: a new look at parasite aggregation. Int. J. Parasitol. 23(7): 937-944.

16. Oliva M, JL Luque and JA Iannacone 1990. The metazoan parasites of Stellifer minor (Tschudi, 1844): An ecological approach. Mem. Inst. Oswaldo Cruz. 85: 271-274.

17. Marcogliese DJ and DK Cone 1996. On the distribution and abundance of eel parasites in Nova Scotia: influence of pH. J. Parasitol. 82: 389-399.

18. Gelnar M, S Sebelova, L Dusek, B Koubkova, P Jurajda and S. Zahradkova 1997. Biodiversity of parasites in freshwater environment in relation to pollution. Parasitologia 39: 189-199.

19. Poulin R 2000. Variation in the intraspecific relationship between fish length and intensity of parasitic infection: biological and statistical causes. J. Fish. Biol. 56(1): 123-137. 
20. Saad-Fares A and C Combes 1992. Abundance/host size relationships in a fish trematode community. J. Helminthol. 66(1): 87-192.

21. Janovy J and EL Hardin 1987. Populations' dynamics of the parasites in Fundulus zebrinus in the Platte river of Nebraska. J. Parasitol. 73: 689-696.

22. Poulin R 1996. Helminth growth in vertebrate hosts: does host sex matter? Int. J. Parasitol. 26: 1311-1315.

23. Rohde K, C Hayward and M Heap 1995. Aspects of the ecology of metazoan ectoparasites of marine fishes. Int. J. Parasitol. 25: 945-970.

(Manuscript received on 18 May, 2013; revised on 20 September, 2013) 